\title{
Endocarditis del marcapasos
}

\author{
R. LÓPEZ RODRÍGUEZ, M. RODRÍGUEZ FRAMIL, A. HERMIDA AMEIJEIRAS, \\ F. L. LADO LADO \\ Servicio de Medicina Interna. Complejo Hospitalario Universitario de Santiago de \\ Compostela. Santiago de Compostela. A Coruña
}

\author{
PACEMAKER ENDOCARDITIS
}

\begin{abstract}
RESUMEN
La endocarditis del marcapasos es una complicación poco frecuente pero grave. La forma más frecuente de presentación es como síndrome febril y/o bacteriemia por gérmenes gram positivos. Dos elementos claves para el diagnóstico son los hemocultivos y el ecocardiograma. Se realiza un análisis retrospectivo de los pacientes con endocarditis del marcapasos ingresados en el Servicio de Medicina Interna de nuestro hospital entre 1989-2003. Se incluyeron 6 pacientes. En este estudio la manipulación repetida del sistema y la diabetes mellitus fueron los factores de riesgo más frecuentes. Los microorganismos más frecuentemente implicados fueron los Staphylococcus spp. A pesar de la menor sensibilidad del ecocardiograma transtorácico, en manos expertas puede mejorar su rendimiento, en esta serie se sitúa en el 66\%. Se realizó extracción mediante cirugía extracorpórea e implantación de un nuevo sistema en la misma intervención en todos, sin ningún caso de recidiva y con una mortalidad del $17 \%$.
\end{abstract}

PALABRAS CLAVE: Endocarditis. Marcapasos.

\begin{abstract}
Pacemaker endocarditis is a rare but serious complication of permanent transvenous pacing. The most common presentation is fever syndrom or gram positive bacteremia. For the diagnostic it is important to performed blood cultures and an echocardiography. A retrospective study included the cases of pacemaker endocarditis diagnosed in the Internal Medicine Department of our Hospital between 1989-2003. Six patients were included. Repeated manipulation of the system and diabetes were the most frequent risk factors. The most frequently detected causative microorganisms were Staphylococci. In spite of the low sensitivity of the transthoracic echocardiography in expert hands it can improve, in this series it places in $66 \%$. Surgical treatment with cardiopulmonar bypass and implantation of a new system was performed in the same intervention in all patients. None relapsed and the overall mortality was $17 \%$.
\end{abstract}

KEY WORDS: Endocarditis. Pacemaker.

López Rodríguez, R, Rodríguez Framil M, Hermida Ameijeiras A, Lado Lado FL. Endocarditis del marcapasos. An Med Interna (Madrid) 2006; 23: 428-430.

\section{INTRODUCCION}

En los pacientes portadores de marcapasos la infección puede afectar al bolsillo del generador, los cables de marcapasos y/o las estructuras cardiacas. Hablamos de endocarditis del marcapasos cuando existen vegetaciones en la porción intravascular del cable y/o su cultivo es positivo. Es una entidad poco frecuente, recientemente se ha estimado una incidencia anual de endocarditis de marcapasos de 1,83 casos por millón de habitantes y 390 casos por millón de portadores de marcapasos y la incidencia de endocarditis, independientemente de la localización, en 2,59 casos por millón de habitantes y 550 casos por millón de portadores de marcapasos (1).

La endocarditis del marcapasos presenta una elevada mortalidad cuando es tratada sólo con antibióticos intravenosos, por lo que se recomienda asociar la retirada completa del sistema, cable y generador $(1,13,15)$. La extracción del marcapasos pue- de realizarse mediante técnicas percutáneas o cirugía. La intervención quirúrgica debe considerarse cuando tiene dos o más cables, lesiones tricúspides, marcapasos con más de un año y vegetaciones grandes $(2,4,8)$. Presentamos una serie de 6 endocarditis del marcapasos en los que se retiró el cable mediante cirugía y se reimplantó, en el mismo acto, un sistema epicárdico.

\section{CASOS APORTADOS}

Análisis retrospectivo de las endocarditis en portadores de marcapasos entre enero de 1989 y diciembre de 2003. Todos los casos fueron reevaluados según los criterios de Duke para el diagnóstico de endocarditis, siendo clasificados como endocarditis definitiva, posible o rechazada $(4,5)$.

Se definió endocarditis del marcapasos definitiva como la presencia de vegetaciones en al menos un cable mediante ecocardiogra-

Trabajo aceptado: 6 de marzo de 2006 
fía transtorácica o transesofágica o si el cultivo del cable intravascular era positivo. Se consideró endocarditis precoz cuando había pasado menos de 2 meses desde la implantación y/o manipulación del sistema y tardía cuando había pasado más de 2 meses.

Se recogieron la edad, sexo, factores de riesgo, características clínicas, resultados microbiológicos, estudios ecocardiográficos, tratamiento y evolución.

Durante el periodo del estudio se diagnosticaron a 8 portadores de marcapasos de endocarditis, de los cuales uno no se incluye en este estudio por no cumplir los criterios de Duke. Un caso presentaba endocarditis valvular protésica aórtica y seis endocarditis del marcapasos, que fueron clasificadas cinco como definitivas y una como posible ya que presentaba bacteriemia persistente por S. epidermidis sin otro foco. Las características de los casos con endocarditis del marcapasos se describen en la tabla I.

La edad media fue de 71 años (rango 63-79 años). Cinco eran varones y dos mujeres. La forma más común de presentación fue como endocarditis tardía y el síntoma más frecuente la fiebre. Los hemocultivos fueron positivos en el $85,7 \%$ de los casos, en la endocarditis del marcapasos que resultaron negativos se habían retirado tras iniciar tratamiento antibiótico y se aisló un $S$. aureus en el cultivo de la herida. En los hemocultivos de la endocarditis valvular protésica se identificó un Enterococcus spp. El ETT fue diagnóstico en el caso de endocarditis valvular protésica y en el $66 \%$ de las endocarditis del marcapasos, en 2 casos se objetivó afectación de la válvula tricúspide y en ningún caso de cámaras izquierdas. En todos se realizó la extracción quirúrgica del sistema después haber iniciado el tratamiento antibiótico intravenoso (rango: 8-76 días), con implantación en el mismo acto de un marcapasos epicárdico. En el caso número 1 no se le pudo retirar el sistema completamente y en el postoperatorio desarrolló un síndrome de cava superior. El caso número 5 falleció en el postoperatorio como consecuencia de un shock cardiogénico (mortalidad: 17\%).

\section{DISCUSIÓN}

La endocarditis del marcapasos es una entidad poco frecuente con una incidencia anual de 1,83 casos/millón habitantes y 390 casos/millón portadores de marcapasos (1) y una prevalencia, en las series más recientes, del 0,5 al 5,7\% (2,4-6,10). Los factores de riesgo pueden clasificarse en aquellos relacionados con el marcapasos (colocación complicada, hematoma del bolsillo del generador, manipulaciones repetidas) y los que dependen del huésped (edad, diabetes, fármacos inmunosupresores, IRC, neoplasias) (1-8,12,14-16). En nuestra serie la mayoría de los casos presentaban algún factor de riesgo, siendo los más frecuentes las manipulaciones múltiples y la diabetes mellitus.

Los microorganismos más frecuentemente implicados son los Staphylococcus spp., responsables de más del 70\% de los casos (1-8,10-14). Otros gérmenes causales son Streptococcus spp., Enterococcus spp., bacilos gram negativos y hongos, fundamentalmente Candida spp. (1-7,10-13). En nuestra serie el $66 \%$ de los casos fueron producidos por Staphylococcus spp y confirmando lo descrito en la literatura, en la endocarditis del marcapasos precoz el agente más frecuente fue el $S$. aureus y en la tardía el $S$. epidermidis $(1-6,8,10)$. En los pacientes con marcapasos y prótesis valvular el germen más frecuente es Streptococcus spp. y Staphylococcus spp. sólo son responsables del $18,2 \%$ de los casos (1). En el caso que notificamos la infección fue producida por un Enterococcus spp.

La endocarditis del marcapasos se puede clasificar como precoz o tardía en función del tiempo transcurrido desde la implantación y/o la última manipulación. La forma precoz representa aproximadamente un tercio de los casos, suele manifestarse en las dos primeras semanas con fiebre y signos inflamatorios locales $(1,4,5,7,9,10)$. La forma tardía es la más frecuente, generalmente tiene un curso subagudo, y se presenta como un síndrome febril sin foco $(1,5-7)$ y/o bacteriemia por gérmenes gram positivos (9). Nuestros pacientes consultaron todos por síndrome febril y sólo uno tenía signos inflamatorios sobre la bolsa del generador. La concurrencia de esplenomegalia, nuevo soplo cardíaco o fenómenos vasculares embólicos sistémicos es rara $(3,6,7)$. No obstante, se producen embolias pulmonares en el 30 al $40 \%$ de los casos que generalmente son poco sintomáticas y bien toleradas $(1,2,4,7,10)$, que algunas veces son la forma de presentación como ocurrió en uno de nuestros casos. La baja frecuencia de fenómenos vasculares embólicos del lado izquierdo puede ser explicada por el hecho de que la infección afecta predominantemente al corazón derecho $(6,7)$.

Está descrito en la literatura que el ETE es la prueba de imagen de elección para el diagnóstico de la endocarditis del marcapasos (2-5,7-11,14-17). El ETT presenta una sensibilidad menor del $30 \%(2,4-7,10,11)$, que en alguna serie llega al 54\% (6),

TABLA I

CARACTERÍSTICAS DE LOS PACIENTES CON ENDOCARDITIS DEL MARCAPASOS

\begin{tabular}{|c|c|c|c|c|c|c|}
\hline & Caso 1 & Caso 2 & Caso 3 & Caso 4 & Caso 5 & Caso 6 \\
\hline Edad & 63 & 70 & 79 & 73 & 65 & 79 \\
\hline Factor de riesgo & Manipulaciones & IRC & No & Manipulaciones & DM, catéter & $\mathrm{DM}$ \\
\hline Síntomas & Fiebre & $\begin{array}{l}\text { Fiebre, supuración } \\
\text { en herida quirúrgica }\end{array}$ & $\begin{array}{l}\text { Fiebre, embolismo } \\
\text { pulmonar }\end{array}$ & Fiebre & Fiebre & Fiebre \\
\hline Precoz/Tardía & Tardía & Precoz & Tardía & Tardía & Tardía & Tardía \\
\hline Hemocultivos & Corynecbacterium & Negativos & $\begin{array}{l}\text { S. coagulasa } \\
\text { negativo }\end{array}$ & S. epidermidis & $\begin{array}{l}\text { S. aureus } \\
\text { meticilin-resistente }\end{array}$ & S. agalactiae \\
\hline ETT/ETE & $\mathrm{ETT}:+$ & $\begin{array}{l}\text { ETT: - } \\
\text { ETE: + }\end{array}$ & $\mathrm{ETT}:+$ & $\begin{array}{l}\text { ETT: - } \\
\text { ETE: - }\end{array}$ & $\mathrm{ETT}:+$ & ETT: + \\
\hline Evolución & $\begin{array}{l}\text { Trombosis de cava } \\
\text { Curación }\end{array}$ & Curación & Curación & Curación & Exitus & Curación \\
\hline
\end{tabular}

IRC: Insuficiencia renal crónica. DM: Diabetes mellitus. ETT: Ecocardiograma transtorácico. ETE: Ecocardiograma transesofágico. 
mientras que el ETE diagnostica aproximadamente el 95\% de los casos $(2,4-6,11)$. Su superioridad se fundamenta en que no se afecta tanto por las reverberaciones del cable, permite visualizar todo el sistema desde la cava superior a la aurícula derecha, así como medir con exactitud el tamaño de las vegetaciones $(16,17)$. No obstante, la ausencia de vegetaciones en el ETE no excluye el diagnóstico de endocarditis y en los casos con elevada sospecha clínica se recomienda repetirlo al cabo de una semana $(11,17)$. Llama la atención en nuestra serie la elevada sensibilidad del ETT, que objetivó la presencia de vegetaciones en el $66 \%$ de los casos, en tres casos sobre el cable y en otro en la aurícula derecha. Este porcentaje es ligeramente superior al 54\% notificado por Arber et al (6) y podría explicarse por el hecho de que en nuestro centro la ecocardiografía es realizada por cardiólogos especializados en esta técnica. En las diferentes series se describe afectación de la válvula tricúspide en el 17 al 32\% $(1,2,4,6-8,18)$ y del corazón izquierdo en el 7 al $18,2 \%$ de los casos $(1,8,11)$. En nuestra serie se objetivó afectación tricúspide en dos casos y no se evidenció afectación de cámaras izquierdas.

El tratamiento de elección consiste en la administración parenteral de antibióticos, durante 4 a 6 semanas, y la retirada del marcapasos, generador y cables, ya que el tratamiento conservador se asocia con una mayor mortalidad y más riesgo de recidiva (1-15). En los portadores de marcapasos casos con bacteriemia, cuando el ETE es normal y no hay signos de infección local, algunos autores recomiendan el tratamiento médico prolongado ya que no se observa un aumento de la morbimortalidad $(1,6,10)$. Sin embargo, Chamis y cols. (9) indican la retirada del marcapasos en las bacteriemias por S. aureus, debido su elevada asociación con endocarditis, cuando hay evidencia clínica o ecocardiográfica de endocarditis, no hay evidencia de endocarditis pero no se identifica la fuente de la bacteriemia y si recurre la bacteriemia tras el tratamiento.

La extracción del sistema puede realizarse mediante técnicas percutáneas o cirugía. La intervención quirúrgica debe considerarse cuando hay dos o más cables, lesiones tricúspides, marcapasos con más de un año y vegetaciones grandes $(2,4,8)$.
La extracción mediante técnicas percutáneas tiene como principales complicaciones la lesión de estructuras cardíacas y la embolización de las vegetaciones $(4,11,13)$. En un principio se consideraron las vegetaciones $>10 \mathrm{~mm}$ una contraindicación relativa, ya que se creía que aumentaban el riesgo de embolismos pulmonares (4). Posteriormente varios autores realizaron la extracción de vegetaciones de hasta $23 \mathrm{~mm}$ sin un incremento de las complicaciones $(2,4,11)$. Meier-Ewert y cols. (13) comunican que aunque las embolias son más frecuentes en las vegetaciones mayores de $15 \mathrm{~mm}$, estas no afectan a la supervivencia ni prolongan el ingreso hospitalario.

La extracción quirúrgica se realiza mediante toracotomía y circulación extracorpórea y tiene menor riesgo de dañar las estructuras cardiacas y de embolización (4). Se asocia a una mortalidad del 12,5 al $21 \%(4,6,7)$ que en alguna serie llega al $40 \%$ (2), aunque también es cierto que generalmente se operan a pacientes con vegetaciones de mayor tamaño y en peor estado general. En nuestra serie, en todos los casos, se realizó la extracción mediante cirugía y se implantó un marcapasos epicárdico en el mismo acto. La infección no recidivó en ningún caso, un paciente falleció en el postoperatorio por shock cardiogénico (mortalidad 17\%) y en otro procedimiento no fue posible la retirada completa del material y en el postoperatorio se produjo una trombosis de la vena cava.

El marcapasos se reimplanta, en el lado contralateral, cuando el paciente está afebril y lleva al menos 10 días sin bacteriemias tras iniciar el tratamiento antibiótico, que se mantiene durante 2 a 4 semanas $(1,3,4,6,7,10,12)$. Otra opción es colocar un sistema epicárdico, que suele utilizarse cuando la extracción es mediante cirugía (10), o un marcapasos temporal externo (3), que es preferible reservarlo para los pacientes inestables ya que tiene un riesgo elevado de reinfección.

En resumen la endocarditis sobre marcapasos es una entidad clínica que debe tenerse en cuenta en todo paciente portador de marcapasos que consulte por fiebre siendo dos elementos claves la realización de hemocultivos y de ecocardiograma.

\section{Bibliografía}

1. Duval X, Selton-Suty C, Alla F, Salvador-Mazen M, Bernard Y, Weber $\mathrm{M}$, et al. Endocarditis in patients with permanent pacemaker: a 1-year epidemiological survey on infective endocarditis due to valvular and /or pacemaker infection. Clin Infect Dis 2004; 39: 68-74.

2. Del Río A, Anguera I, Miró JM, Mont L, et al. Surgical treatment of pacemaker and defibrillator lead endocarditis. Chest 2003; 124: 1451-9.

3. Karchmer AW, Lonworth DL. Infections of intracardiac devices. Cardiol Clin 2003; 21: 253-71.

4. Klug D, Lacroix D, Savoye C, et al. Systemic infection related to endocarditis on pacemaker leads: clinical presentation and management. Circulation 1997; 95: 2098-107.

5. Baños R, Gómez J, Sánchez B, De la Morena G, et al. Pacemaker lead endocarditis: analysis of 11 cases. Enferm Infecc Microbiol Clin 2000; 18: $267-70$.

6. Aber N, Pras E, Copperman Y, Schapiro JM, Meiner V, Lossos IS, et al. Pacemaker endocarditis. Report of 44 cases and review of the literature. Medicine 1994; 73: 299-305.

7. Cacoub P, Leprince P, Nataf P, Hausfater P, Dorent R, Wechsler B, et al. Pacemaker infective endocarditis. Am J Cardiol 1998; 82: 480-4.

8. Voet JG, Vandekerckhove YR, Muyldermans L, Missault LH, Matthys LJ. Pacemaker lead infection: report of three cases and review of the literature. Heart 1999; 81: 88-91.

9. Chamis AL, Peterson GE, Cabell CH et al. Staphylococcus aureus bacteriemia in patients with permanent pacemakers or implantable cardio- verter-defibrillators. Circulation 2001; 104: 1029-33.

10. Dumont E, Camus C, Victor F, et al. Suspected pacemaker or defibrillator transvenous lead infection. Prospective assessment of TEE-guided therapeutic strategy. Eur Heart J 2003; 24: 1779-87.

11. Victor F, De Place C, Camus C, et al. Pacemaker lead infection: echocardiographic features, management and outcome. Heart 1999; 81: 82-7.

12. Chua JD, Wilkoff BL, Lee I, et al. Diagnosis and management of infections involving implantable electrophysiologic devices. Ann Intern Med 2000; 133: 604-8

13. Meir-Ewert HK, Gray ME, John RM. Endocardial pacemaker or defibrillator leads with infected vegetations: a single-center experience and consequences of transvenous extraction. Am Heart J 2003; 146: 339-44.

14. Remacha Esteras MA, Esteban Martín A, Herrera Rubio JA. Staphylococcus schleiferi. An Med Interna (Madrid) 2004; 21: 68.

15. Sánchez Sierra B, Baños Madrid R, Andreu J, García-Estan J, Gómez Gómez J. Endocarditis caused by pacemaker lead. An Med Interna (Madrid) 2002; 19: 100-1.

16. Rallidis LS, Komninos KA, Papasteriadis EG. Pacemaker-related endocarditis: the value of transesophageal echocardiography in diagnosis and treatment. Acta Cardiol 2003; 58: 31-4.

17. San Román JA, Vilacosta I. Role of transesophageal echocardiography in right-sided endocarditis. Echocardiography 1995; 12: 669-72.

18. Climent Payá V, Martínez Martínez JG, Marín Ortuno F, et al. Natural-valve endocarditis in patients wearing pacemakers. Rev Clin Esp 1999: 28-30. 